\title{
TopicExplorer: Exploring Document Collections with Topic Models
}

\author{
Alexander Hinneburg, Rico Preiss, and René Schröder \\ Informatik, Martin-Luther-University Halle-Wittenberg, 06099 Halle, Germany \\ hinneburg@informatik. uni-halle.de, \\ \{rico.preiss, rene.schroeder\}@student.uni-halle.de
}

\begin{abstract}
The demo presents a prototype - called TopicExplorer- that combines topic modeling, key word search and visualization techniques to explore a large collection of Wikipedia documents. Topics derived by Latent Dirichlet Allocation are presented by top words. In addition, topics are accompanied by image thumbnails extracted from related Wikipedia documents to aid sense making of derived topics during browsing. Topics are shown in a linear order such that similar topics are close. Topics are mapped to color using that order. The auto-completion of search terms suggests words together with their color coded topics, which allows to explore the relation between search terms and topics. Retrieved documents are shown with color coded topics as well. Relevant documents and topics found during browsing can be put onto a shortlist. The tool can recommend further documents with respect to the average topic mixture of the shortlist.
\end{abstract}

Keywords: topic model, document browser.

URL: http://topicexplorer.informatik.uni-halle.de (Firefox 13 or later with JavaScript)

\section{Introduction}

The exploration of large unstructured text collections pose a difficult problem for humans in general. Search engines offer fast access to documents via keyword search, which, however, requires to know what you are searching. Therefore, search engines are not the perfect tool to explore the unknown in document collections.

Topic models may assist such an exploration by offering ordered words lists that are often recognized as general semantic topics present in the document collection. The inference algorithm estimates a set of probability distributions (topics) over the vocabulary of unique words.

A major problem is to assess the interpretability of found topics [1. In order to develop such methods, researchers need to get an intuition about the results of topic modeling. This can be done by tracing back topics as well as document specific topic mixtures to the original documents to check how inferred topics and possible interpretations of them do appear there. Because of the sizes of 
a typical topic model with hundreds of topics and a document collection with thousands of documents, this is impossible to do by hand.

We set up a novel topic browser - called TopicExplorer- that helps to inspect topics and facilitates to develop and check interpretations of the found topic distributions. For these purposes, TopicExplorer combines topic modeling, key word search and visualization to give the user an intuition about the strengths and drawbacks of the computed topics.

\section{Demonstration of TopicExplorer}

The underlying data for our demonstration is the subset of the English Wikipedia that contains all pages in the general categories person or place. Without page forwards, the document collection contains 716,874 documents in total. We build an LDA topic model [2] using MALLET [3] having 200 topics.

TopicExplorer is a web-based application with a MySQL back-end that stores data of the document collection as well as the topic model. The GUI (see Figure1] top) shows all topics as a horizontal row of colored boxes at the bottom. Each box shows the list of most important words of a topic that are ordered by decreasing probability of a word given a topic $p(w \mid z)$. The font size is also proportional to this probability and indicates how fast the probabilities are decreasing. In addition to the words, each topic in accompanied by a list of associated images that can be scrolled by clicking on the smaller left or right thumbnails. Images are associated to a topic $z$ if many words in the neighborhood of the image link in the wiki-text are probabilistically assigned to $z$. Top words as well as images of a topic are shown as full screen overlay when the lens icon of topic is clicked (see Figure 1 middle). Viewing images and words together helps to find an interpretation of the topic.

The topics are ordered by similarity into a linear list that can be horizontally scrolled using the slider (gray box) below the color bar at the very bottom of the GUI. The ordering is derived using Wards hierarchical clustering as implemented in $\mathrm{R}$ that takes cosine similarities the between topic distributions as input and outputs a layout of the dendrogram. That layout implies an ordering of the leafs, which are the topics in this case. The ordering is quite helpful to organize the large topic set, e.g. the screen-shot (Figure 1 top) shows different team sports (football, hockey, soccer and baseball) close together. The linear order of topics is used to map topics to colors (the rainbow color-map in this demo). Topic color is used to indicate the most important topics of a document in the respective snippets. By hovering with the mouse over such a colored circle shows a tool tip with the three most important words, e.g. Figure 1 bottom left. Clicking a circle takes the user directly to the topic. A similar visualization is used to indicate the most important topics of a word in the auto-completion when typing a search keyword (see Figure 1 bottom right). There, topics are ordered by decreasing $p(z \mid w)$. The visualization in the autocompletion helps to identify ambiguous words, like football.

Topics as well as documents can be added to a shortlist at the right of the screen (Figure 1 top). Using the Recommend-Button, the average topic mixture 

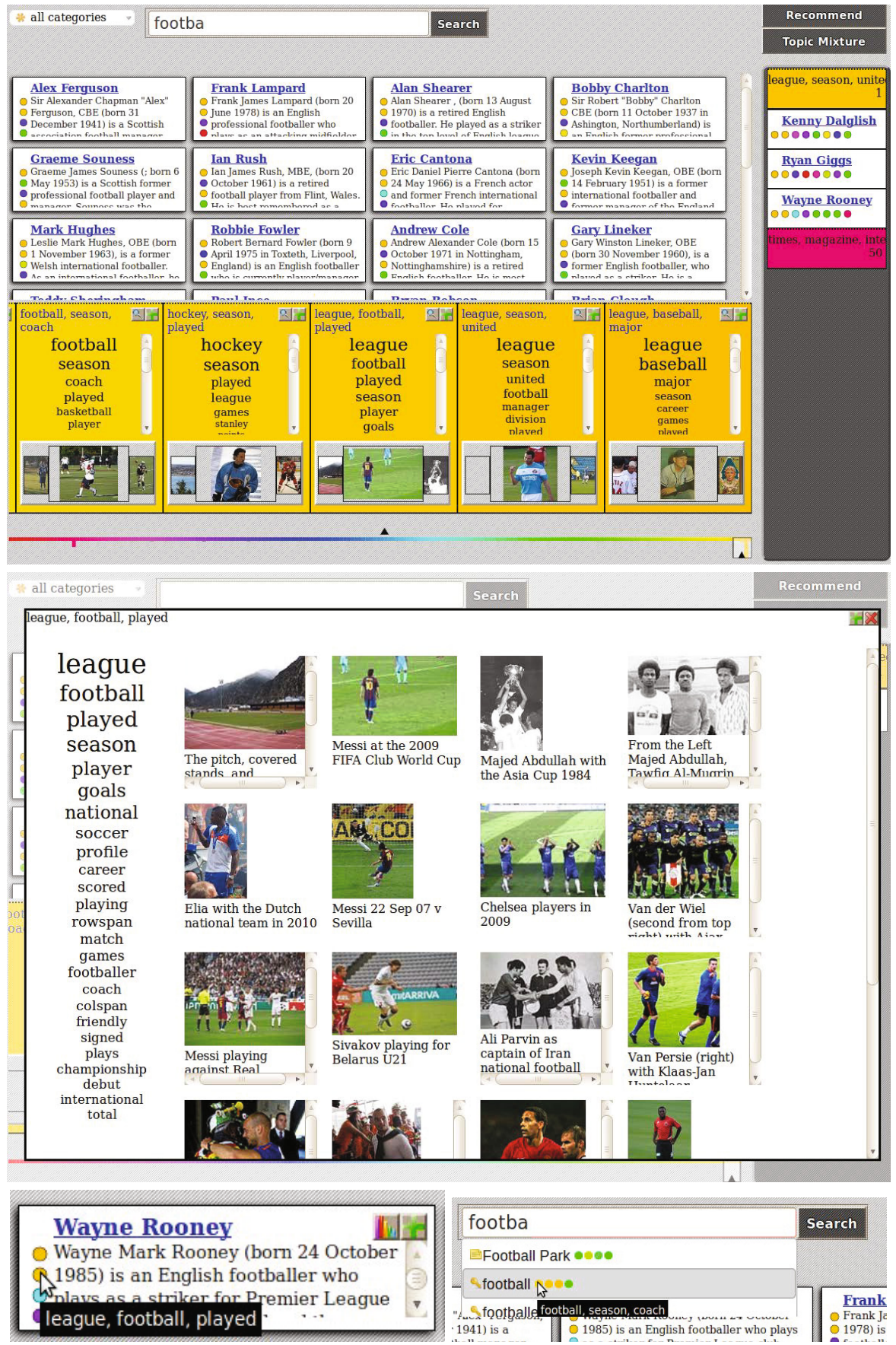

Fig. 1. TopicExplorer 
distribution of topics and documents in the shortlist is used as a cosine similarity search query to find documents with similar topic mixture distribution. We use LSH [4] to make this search of the whole document collection fast enough for an interactive application. This similarity search helps to trace the topics back to the original documents that define the topic. Keyword search completes the GUI, which helps the user to focus the evaluation of the topic model onto a specific area of interest.

\section{Related Work}

Related software projects include the Topic Model Visualization Engine by Chaney and Blei (http://code.google.com/p/tmve/) that transforms a topic model into a precomputed set of web pages. It shows important words for each topic as well as related documents and related topics. The Topical Guide (https://facwiki.cs.byu.edu/nlp/index.php/Topical_Guide) shows similar informations for each topic. In addition, it allows to filter by topics, different metrics, words and documents and can produce parallel coordinates plots that relate topics with other meta-data. The closest match to our TopicExplorer is the topic-based search interface SearchInaBox to Wikipedia by Buntine (http://wikipedia.hiit.fi/H100topiclist.html). It allows to search documents by keywords and filters optionally afterwards by topical text. The result list shows the important topics of the documents. Additionally to the search engine functionality, it offers a topic browser that shows important words and documents for each topic.

Our TopicExplorer is different from all these tools by showing images related to topics to aid intuitive interpretation of topics. Furthermore, it is the only one that allows similarity search of the document collection by arbitrary topic mixture distributions.

Acknowledgements. We thank R. Fildebrandt, T. Gottlieb, T. Nguyen, M. Pfuhl, M. Kunze and C. Wangemann for their contributions to the code base of TopicExplorer as well as A. Both of Unister $\mathrm{GmbH}$ for helpful discussions.

\section{References}

1. Blei, D.M.: Probabilistic topic models. Commun. ACM 55(4), 77-84 (2012)

2. Blei, D.M., Ng, A.Y., Jordan, M.I.: Latent dirichlet allocation. J. Mach. Learn. Res. 3, 993-1022 (2003)

3. McCallum, A.K.: Mallet: A machine learning for language toolkit (2002), http://mallet.cs.umass.edu

4. Charikar, M.S.: Similarity estimation techniques from rounding algorithms. In: Proc. of STOC 2002, pp. 380-388. ACM (2002) 